\title{
Introduction: A Special Issue on Cardiovascular Development and Disease
}

According to statistics provided by the American Heart Association for 2002, the latest year available, cardiovascular diseases are the leading cause of death in both males and females in the United States with over 434,000 males and 494,000 females dying each year from cardiovascular-related diseases. This is $36.6 \%$ of all deaths in males and $39.8 \%$ in females and represents billions of dollars in health care costs. Cardiovascular-associated diseases run the gamut from congenital defects that manifest early in development through diseases such as stroke and infarcts frequently associated with advanced age. Investigative techniques to study these diseases are varied and include a full range of imaging, genomic, and proteomic technologies.

Among the techniques used to study the development and diseases of the cardiovascular system, microscopy is one of the oldest and has been used since the early advent of magnifying optics. This issue includes a series of invited articles that were presented at the Microscopy and Microanalysis 2004 meeting that was held in Savannah, Georgia, from August 1 through August 4, 2004. Although they represent a very small sampling of imaging applications in the study of the cardiovascular system, they do illustrate the variety of the types of studies that can be performed. They also present an array of new data collected from in vitro and in vivo experiments designed to examine normal development and pathological events that involve the heart and its associated vasculature.

In a brief review, Borg et al. touch on the history of microscopy in studying the cardiovascular system by noting that early studies imaged the flow of blood cells through the vasculature of thin mesenteries. They go on to illustrate the variety of information that traditional imaging techniques such as light microscopy and scanning and transmission electron microscopy have provided over the years. These authors also provide information on other types of imaging that are now available such as ultrasound, magnetic resonance imaging (MRI), positron emission tomography (PET), and others that have become important in examining the structure and function of the heart. The article concludes with an illustration of how the merging of disciplines such as biology, medicine, and engineering will be required if the mechanical properties of actively transforming heart tissues are to be fully understood.

Two of the articles present improvements in microscopy technology for the study of the cardiovascular system. Planck and Sussman address an important problem in understanding the physiology of cardiac myocytes by describing methodology to directly measure intracellular $\mathrm{Ca}^{2+}$ levels using inactivated, Fluo-3 AM loaded cardiomyocytes equilibrated with $\mathrm{Ca}^{2+}$ concentration standards. Determination of calcium dynamics in active cardiomyocytes is essential in understanding how diseases that affect beating patterns of the heart disrupt cardiomyocyte function. The article by Miller et al. focuses on the development of techniques for improved confocal imaging and three-dimensional reconstruction of regions of the heart. As with all organs that consist of multiple cell types, developing an understanding of the structural relationships of the various types of tissue components is important, and three-dimensional imaging techniques have been instrumental in generating models of cardiac development and disease.

Articles by Linask et al. and Bullard et al. present data illustrating the role of the extracellular matrix in presenting "outside-in" signaling events that affect the cellular development and differentiation of myocytes in the heart. Linask et al. look at the cross talk between molecules in the adhesion signaling pathways and how environmental insults such as exposure to lithium can affect signaling and result in embryonic lethality 
or fetuses born with severe heart defects. Bullard et al. also examine signaling pathways by looking at the role that different extracellular matrix components play in protein kinase $\mathrm{C}$ signaling and how changes in substrate and signaling affect myocyte growth and differentiation.

Zhu et al. address a second important aspect of cell communication within the heart by characterizing the colocalization of proteins that function in cell:cell communication between neonatal cardiomyocytes. By the use of multiple labeling techniques and confocal microscopy, they describe the expression patterns and levels of colocalization between $\mathrm{ZO}-1$ and $\mathrm{Cx} 43$, two proteins that are found in gap junctions that function in cellular communication.

Whereas the above articles focus on cardiac myocytes, the article by Morales et al. examines the distribution of discoidin domain receptor 2 (DDR2) in the developing heart. In previous work this group has shown that in adult hearts DDR2 distribution is limited to fibroblasts, but the distribution of DDR2 and fibroblasts during heart development have not been adequately described. Using confocal microscopy, these authors have found that initially, DDR2 expression can be found on the epicardial surface of the heart and on endothelial and mesenchymal cells within the cardiac cushions. By the use of this fibroblast specific marker, they then describe the spatial distribution of fibroblasts throughout the development of the heart.

A major problem in using culture techniques to study cardiovascular development and disease is that cardiac myocytes and fibroblasts change phenotype when isolated from the heart and placed in culture. Several groups have attempted to develop culture techniques that maintain an in vivo phenotype when these cells are placed in culture. Camelliti et al. describe their use of microfluidic techniques to grow two-dimensional structured cardiac tissue models, containing both myocytes and fibroblasts, and characterize cell morphology, distribution, and cell:cell coupling using immunohistochemical techniques. In vitro findings were compared with in vivo ventricular cyto-architecture, illustrating that cardiac myocytes and fibroblasts, cultured on intersecting $30-\mu \mathrm{m}$-wide collagen tracks, acquire an in vivo-like phenotype.

Articles by Sedmera et al. and Jerome et al. examine in vivo aspects of cardiovascular function. Sedmera et al. use an optical mapping approach to demonstrate the patterns of electrical activation in embryonic hearts that result in a functional beating pattern and examine the role of hemodynamic loading in the developmental patterns of the activation. The final article in the issue, by Jerome et al., addresses the ever increasing problem in the adult population of coronary artery thrombosis. Using confocal microscopy they examine how thrombolytic clots form and mechanisms that may potentially inhibit their formation.

Although obviously not all inclusive of how microscopy and other imaging techniques are being used to study cardiovascular development and disease, the above sampling of research is an excellent example of the role imaging plays in this important field. As technology continues to improve the imaging techniques in the basic and clinical sciences, we will continue to further our understanding of the normal development and disease processes in the circulatory system.

Robert L. Price

University of South Carolina

School of Medicine

Guest Editor 